\title{
NUEVOS HAMMATOCERATINAE (AMMONITINA) DEL LÍMITE AALENIENSE/BAJOCIENSE DE LA ZONA SUBBÉTICA (CORDILLERA BÉTICA, SUR DE ESPAÑA)
}

\author{
Asunción LINARES
}

Departamento de Estratigrafía y Paleontología, Facultad de Ciencias, Universidad de Granada, E-18002, Granada

Linares, A. 2002. Nuevos Hammatoceratinae (Ammonitina) del límite Aaleniense/Bajociense de la Zona Subbética (Cordillera Bética, sur de España). [New Hammatoceratinae (Ammonitina) from the Aalenian/Bajocian boundary of the Subbetic Zone (Betic Cordillera, southern Spain).] Revista Española de Paleontología, 17(2), 285-291. ISSN 0213-6937.

\begin{abstract}
The Hammatoceratinae from the Aalenian/Bajocian boundary beds of the Cortijo Despeñadero section, Montillana (province of Granada, Betic Cordillera), are analysed. Several specimens correspond to hitherto undescribed species. Morphological characters of these forms are typical of the subgenus Eudmetoceras (Eudmetoceras). A new species, E. (E.) subbeticum n. sp., and a possible new species, E. (E.) sp. 1 aff. $E$. (E.) subbeticum, are for the first time here described. E. (E.) subbeticum includes Hammatoceratinae with wide and shallow umbilicus, strong, radial or slightly retroverse ribs and scarcely developed tubercles. $E$. (E.) sp. 1 aff. $E$. (E.) subbeticum is similar to $E$. (E.) subbeticum, but is more involute and has flatter flanks.
\end{abstract}

Keywords: Ammonitina, Hammatoceratidae, new species, Middle Jurassic, Subbetic, southern Spain.

\section{RESUMEN}

Se analizan varios ejemplares de Hammatoceratinae correspondientes a formas nuevas procedentes del Aaleniense Superior-Bajociense inferior de la sección del Cortijo del Despeñadero, Montillana (provincia de Granada, Cordillera Bética). La morfología de las mismas nos permite incluirlas en el subgénero Eudmetoceras (Eudmetoceras). Una especie nueva, E. (E.) subbeticum n. sp., y otra posiblemente también nueva, $E$. (E.) sp. 1 aff. E. (E.) subbeticum, se describen por primera vez en este trabajo. E. (E.) subbeticum comprende hammatoceratinos de ombligo amplio y poco profundo con costillas radiales o ligeramente retroversas, provistas de tubérculos poco desarrollados. $E$. (E.) sp. 1 aff. $E$. (E.) subbeticum es muy similar a $E$. (E.) subbeticum, pero es más involuto y tiene los flancos más planos.

Palabras clave: Ammonitina, Hammatoceratidae, especie nueva, Jurásico Medio, Subbético, sur de España.

\section{INTRODUCCIÓN}

Durante los diversos muestreos realizados en el área del Cortijo del Despeñadero entre las décadas de los años setenta y ochenta del pasado siglo se recogieron gran cantidad de ammonites de edad Aaleniense superiorBajociense inferior, cuyas asociaciones están dominadas por formas pertenecientes fundamentalmente a las familias Graphoceratidae (Graphoceras, Ludwigella, Hyperlioceras y Braunsina) y Erycitidae (Haplopleuroceras). Aparte de estos taxones dominantes, otras formas como Grammoceratinae (Asthenoceras y Fontannesia), Haploceratidae (Lissoceras y Bradfordia), Otoitidae (Riccardiceras),
Sonniniidae (Euhoploceras y Nannoceras), Hammatoceratidae [Eudmetoceras (Eudmetoceras), Eudmetoceras (Rhodaniceras), Euaptetoceras)] así como Phylloceratina (Phylloceras, Holcophylloceras y Calliphylloceras) y Lytoceratina (Lytoceras y Alocolytoceras) son también elementos comunes. En este trabajo se analizan algunos ejemplares pertenecientes al género Eudmetoceras (Hammatoceratidae, Ammonitina) del límite Aaleniense/ Bajociense, los cuales no se corresponden con ninguna de las especies de este género hasta ahora descritas y que posiblemente representen dos especies nuevas.

Todos los ejemplares de Eudmetoceras aquí analizados han sido recogidos en muestreos realizados en 


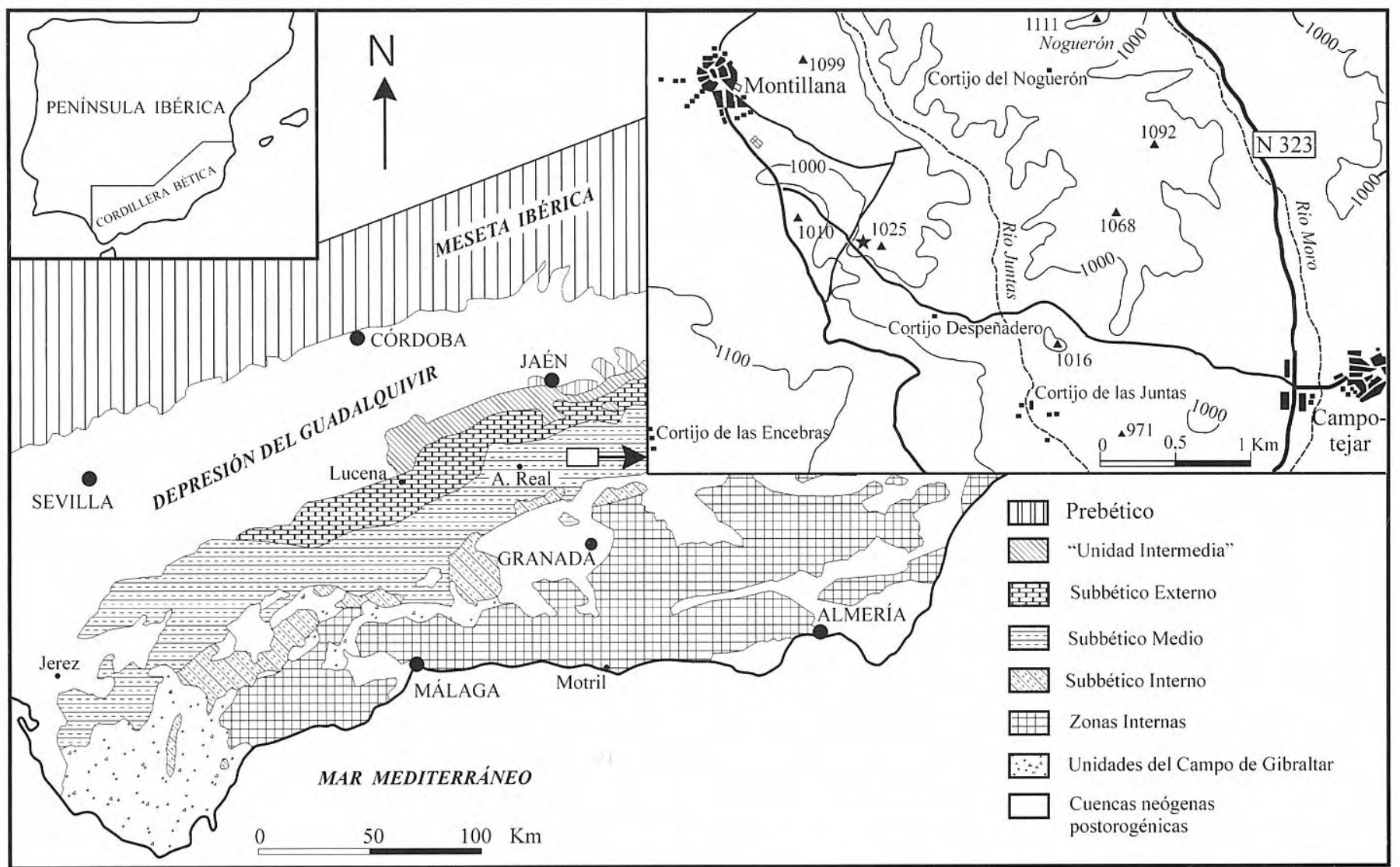

Figura 1. Localización geográfica y geológica de la sección del Despeñadero, Montillana (Granada). La estrella indica la situación exacta de los niveles muestreados.

Geographical and geological location of the Despeñadero section, Montillana (Granada). The star indicates the exact location of the sampled beds.

la sección de Cortijo del Despeñadero. Dicha sección está situada en el sector central del dominio Subbético medio, aproximadamente $1,5 \mathrm{~km}$ al sureste del pueblo de Montillana (Provincia de Granada), junto al margen derecho de la carretera local que comunica Montillana y Campotejar (Fig. 1). En esta localidad los materiales correspondientes al Aaleniense terminal (Zona de Concavum, Subzona de Limitatun) y Bajociense basal (Zona de Discites, Subzona de Walkeri), de donde proceden los ejemplares estudiados, están formados por unos 4,5 metros de margas y margocalizas grises con abundantes ammonoideos (García-Dueñas et al., 1967; Linares y Sandoval 1993). Esta ritmita está limitada a muro y techo por sendas coladas de rocas volcánicas (Fig. 2). Gran parte de los ammonites recogidos en el Despeñadero proceden de derrubios y cantos sueltos, consecuencia de la roturación del terreno para explotaciones agrarias (olivar) y sólo unos cuantos han sido recogidos en los niveles no triturados. Aparte del material aquí analizado, diferentes ammonites (Asthenoceras, Fontannesia, Haplopleuroceras, Euhoploceras y Riccardiceras) procedentes de la misma localidad y de los mismos niveles han sido previamente estudiados como parte de trabajos taxonómicos más amplios (Linares y Sandoval, 1988, 1993, 1996; Sandoval y Chandler 2000; Sandoval et al., 2001).

\section{POSICIÓN TAXONÓMICA DE Eudmetoceras}

ORDEN AMMONOIDEA Zittel, 1884

SUBORDEN AMMONITINA Hyatt, 1889

Superfamilia Hildoceratoidea Hyatt, 1867

Familia Hammatoceratidae Buckman, 1887

Subfamilia Hammatoceratinae Buckman, 1887

Género Eudmetoceras Buckman, 1920

Especie tipo: Eudmetoceras eudmetum Buckman, 1920; T.A. III; Lám. 179.

\section{Descripción}

El género Eudmetoceras comprende especies de hammatoceratinos caracterizadas por tener un enrollamiento evoluto y ombligo amplio y plano. En este género se distinguen dos series de formas: Eudmetoceras (Rhodaniceras), que comprende formas de pequeño tamaño con línea de sutura poco dividida y con peristoma que presenta apófisis yugales bien desarrolladas, y Eudmetoceras (Eudmetoceras) de mayores dimensiones, con línea de sutura más recortada y con peristoma simple. $E$. (Rhodaniceras) presenta además un surco a ambos lados de la carena, carácter que es único en todas las especies comprendidas en este grupo de hammatoceratinos. 


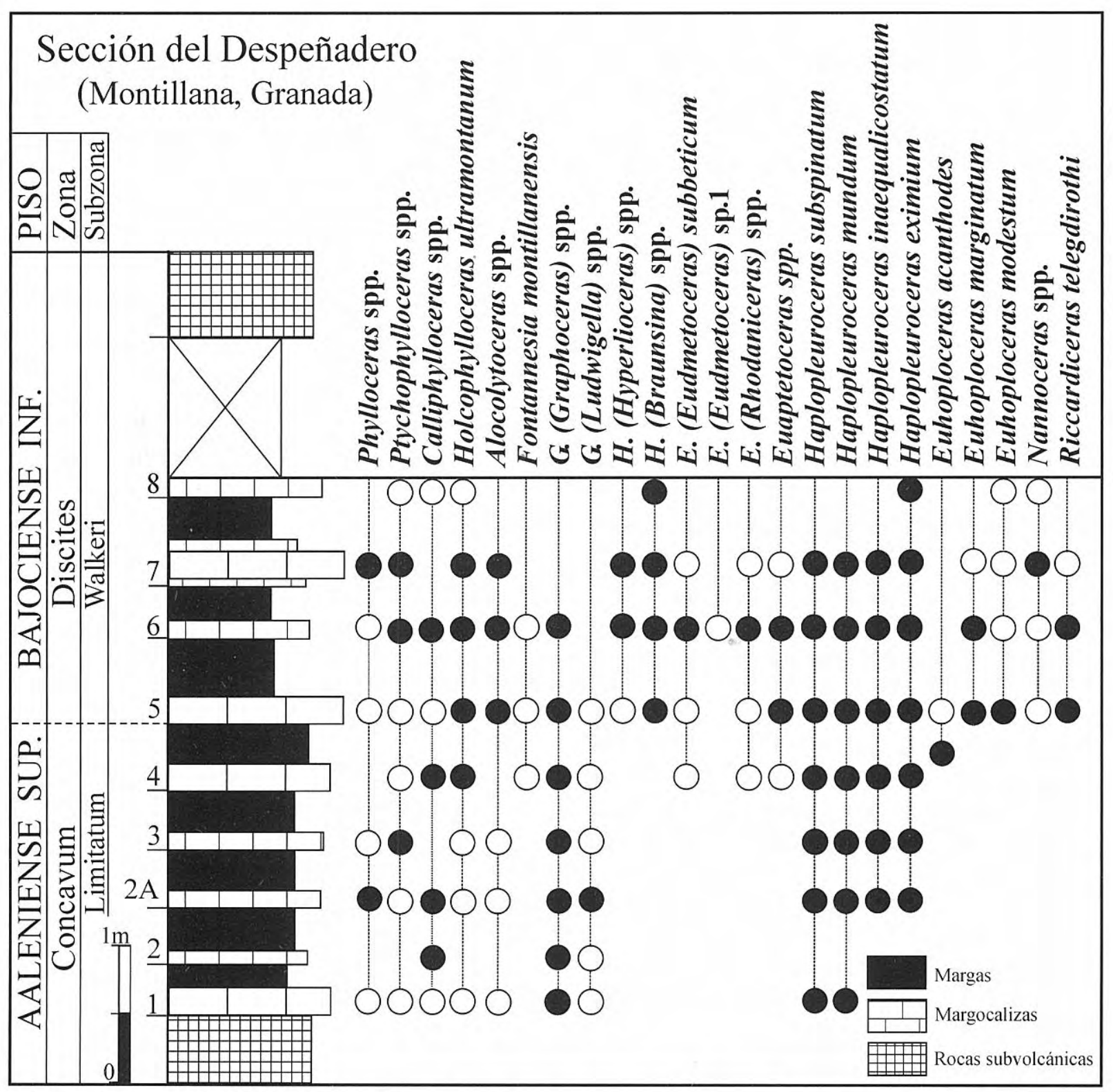

Figura 2. Columna estratigráfica de la sección del Despeñadero, Montillana (Granada) indicando la distribución de especies de ammonites. Los puntos negros indican ejemplares recogidos "in situ". Los círculos se refieren a la posible procedencia de ejemplares sueltos.

Lithological succession and ammonite range in the Despeñadero section, Montillana (Granada). Black points refer to exemplars sampled "in situ", and circles indicate possible origin of the exemplars from loose blocks.

Subgénero Eudmetoceras (Rhodaniceras) Elmi, 1963 [m]

Especie tipo: Hammatoceras subinsigne [(non Oppel, Bayle 1878, lám. 82, fig. 3 = Hammatoceras rhodanicum Renz, 1925 , p. $15=$ Eudmetoceras (Rhodaniceras) rhodanicum (Renz)].

\section{Diagnosis}

De acuerdo con su diagnosis original (Elmi, 1963, p. 60), E. (Rhodaniceras) incluye "Hammatoceratinae de tamaño pequeño, crecimiento lento de la espira, ombligo ancho y plano, vueltas de espira poco recubridoras. La sección, bastante variable, es casi cuadrada entre las costillas o los tubérculos, el borde y el muro umbilical están más o menos marcados según las especies. La región ventral contiene una carena hundida entre dos surcos muy poco marcados. La ornamentación varía de unas especies a otras, pero las costillas permanecen siempre bien marcadas hasta el final de la cámara de habitación. El peristoma presenta dos apófisis anchas y redondeadas en la base. La línea de sutura es de tipo hammatoceratino con lóbulo suspensivo bien pronunciado".

\section{Distribución estatigráfica}

E. (Rhodaniceras) es conocido en el Aaleniense Superior (Zona de Concavum) y en el Bajociense Inferior (Zona Discites). 

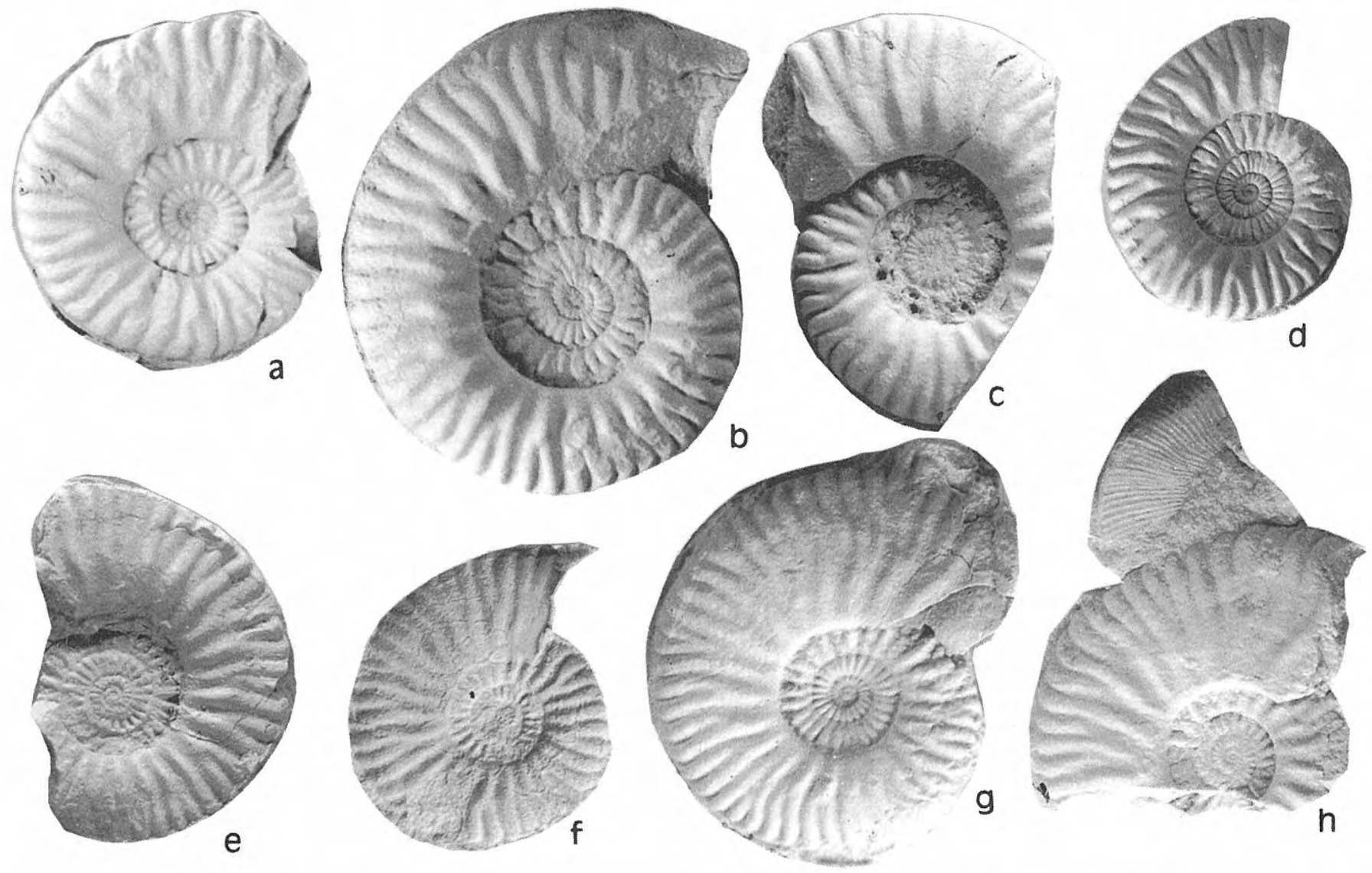

Figura 3. a-e, Eudmetoceras (Eudmetoceras) subbeticum n. sp. [M]: a, Mo.76-1; b, Mo.73-2 (holotipo); c, Mo.81-1; d, Mo.74-1; e, Mo.78-1. f-h, Eudmetoceras sp.1 aff. E. (E.) subbeticum n. sp. [M]: f, Mo.87-3; g, Mo.76-4; h, Mo.782. Todos los ejemplares proceden del Aaleniense terminal o Bajociense basal de la sección del Despeñadero, Montillana (Granada) y están figurados a tamaño natural.

a-e, Eudmetoceras (Eudmetoceras) subbeticum n. sp. [M] a, Mo.76-1; b, Mo.73-2 (holotype); $\boldsymbol{c}$, Mo.81-1; d, Mo.741; $\boldsymbol{e}$, Mo.78-1. 3f-h, Eudmetoceras sp.1 aff. E. (E.) subbeticum n. sp. [M]: 3, Mo.87-3; 3, Mo.76-4; h, Mo.78-2. All specimens, from latest Aalenian or earliest Bajocian of the Despeñadero section, Montillana (Granada), are figured at natural size.

\section{Discusión}

Según Elmi (1963), E. (Rhodaniceras) presenta varios caracteres comunes con $E$. (Eudmetoceras), en particular el enrollamiento lento, el ombligo plano y el aspecto de conjunto de la línea de sutura. Estas semejanzas son suficientes para agruparlos en un mismo género. Aunque en este estudio sólo se han analizado formas macroconchas correspondientes a nuevas especies, en la sección del Cortijo del Despeñadero se han recogido varias formas microconchas que parecen corresponderse con E. (Rhodaniceras) prosphues (Buckman). En algunas de estas formas subbéticas es posible observar la línea de sutura, de tipo hammatoceratino, con lóbulo suspensivo bien pronunciado.

Subgénero Eudmetoceras (Eudmetoceras) Buckman, $1898[\mathrm{M}]$

\section{Diagnosis}

Formas macroconchas de tamaño medio a grande, crecimiento lento de la espira, ombligo ancho y poco profundo, vueltas de espira poco recubridoras. La sección, bastante variable, oscila entre subrectangular comprimida, casi cuadrada y subtriangular en los espacios intercostales; el muro y el reborde umbilical están más o menos marcados. La región ventral contiene una carena elevada limitada por dos bandas lisas. La ornamentación varía de unas especies a otras; las costillas, simples o divididas a altura variable, permanecen bien marcadas hasta el final de ontogenia; aparecen tubérculos o engrosamientos tuberculiformes en el punto de división de las costillas. El peristoma es simple. La línea de sutura es de tipo hammatoceratino con lóbulo suspensivo bien pronunciado.

\section{Distribución estratigráfica}

E. (Eudmetoceras) es conocido en el Aaleniense Superior (Zona de Concavum) y en el Bajociense Inferior (Zona Discites). En la Cordillera Bética aparece en materiales de esta edad en varias secciones del dominio Subbético. 


\section{Discusión}

Entre las especies hasta ahora conocidas pertenecientes al subgénero E. (Eudmetoceras) se encuentran tres especies previamente nominadas procedentes del Tetis occidental: la especie tipo del género, E. eudmetum que procede del Dorset (Inglaterra), E. flexuosum Elmi (1963, p. 40, figs 13-14 en texto, lám. 3, fig. 5) y E. masticonensis Elmi (1963, p. 69, figs 25-26 en texto, lám. 9, fig. 5) procedentes del sureste de Francia. Martínez (1992, lám. 40, figs 2-4; 1996, p. 116) figuró tres fragmentos de Eudmetoceras procedentes de la Cordillera Ibérica, dos de los cuales son totalmente indeterminables (Eudmetoceras sp. 2 y Eudmetoceras sp. 3) y otro (Eudmetoceras sp. 1) es parecido a algunos ejemplares subbéticos, aunque parece tener los tubérculos más desarrollados en vueltas internas. Sadki (1996, pp. 164-165, lám. 3, figs. 4-6) describe y figura algunos ejemplares de E. (Eudmetoceras) procedentes del Atlas Central (Marruecos), los cuales son claramente diferentes de las formas subbéticas.

Además de las especies tetísicas, algunas formas procedentes del Pacífico Oriental han sido atribuidas al subgénero E. (Eudmetoceras): E. (E.) gerthi (Jaworski, 1926), lectotipo en Westermann y Riccardi (1972, lám. 2, fig. 2), E. (E.) jaworskii Westermann (1964, p. 412), lectotipo en Westermann y Riccardi (1972, lám. 2, fig. 3) y E. nucleospinosum Westermann (1964, p. 414, lám. 66, figs. 3-5). Estas formas pacíficas, relativamente involutas y con costillas bastante flexuosas, no parecen tener muchas afinidades con las formas de E. (Eudmetoceras) procedentes del Tetis occidental.

\section{Eudmetoceras (Eudmetoceras) subbeticum $\mathrm{n}$. sp. $[\mathrm{M}]$ \\ Fig. 3a-e}

Derivación del nombre: El nombre hace referencia a la Cordillera Subbética (sur de España), de donde proceden todos los ejemplares disponibles pertenecientes a esta nueva especie.

Holotipo: El ejemplar Mo.73-2, depositado en el museo del Departamento de Estratigrafía y Paleontología de la Universidad de Granada.

Estrato y localidad típicos: Bajociense inferior (Zona de Discites, Subzona de Walkeri), niveles 5 a 8 , sección del Despeñadero, Montillana, Provincia de Granada, España.

Material: Mo.73-2, holotipo, molde interno completo en buen estado de conservación; Mo.74-1, molde interno en buen estado de conservación asociado a Haplopleuroceras eximium Gerar; Mo.74-2, molde interno de un ejemplar juvenil un poco deformado; Mo.76-1, Mo.76-3, Mo.76-3, moldes internos de ejemplares juveniles relativamente bien conservados; Mo.78-1, molde interno bastante aplastado con el muro umbilical vertical; Mo.79-1, molde interno incompleto algo erosionado; Mo.81-1, molde interno incompleto con las vueltas internas muy aplastadas, muy parecido al holotipo, pero de tamaño más reducido; Mo.841, fragmento no deformado; Mo.84-2, fragmento de un molde interno muy similar al holotipo; Mo.87-1, molde externo incompleto y algo deformado, Mo.87-2, ejemplar muy deformado; Mo.88-1, ejemplar juvenil bastante aplastado; MoD.5.1, fragmento no muy bien conservado, muy similar al holotipo.

\section{Dimensiones en mm}

$\mathrm{D}=$ diámetro del ejemplar; $\mathrm{O}=$ diámetro umbilical; $\mathrm{H}=$ altura de la espira; $\mathrm{N} 1$ = número de costillas primarias por vuelta de espira; $\mathrm{N} 2$ = número de costillas secundarias por vuelta de espira

$\begin{array}{lccccccc}\text { ejemplar } & \mathbf{D} & \mathbf{O} & \mathbf{O} / \mathbf{D} & \mathbf{H} & \mathbf{H} / \mathbf{D} & \mathbf{N 1 / 2} & \mathbf{N} 2 / 2 \\ \text { M0.73-2 } & 65,0 & 27,1 & 0,42 & 23,9 & 0,37 & 12,0 & 23,0 \\ - & 49,5 & 21,4 & 0,43 & 17,2 & 0,34 & 12,5 & 21,0 \\ \text { Mo.74-2 } & 40,0 & 17,5 & 0,44 & 13,2 & 0,33 & 12,5 & 21,0 \\ - & 32,5 & 15,0 & 0,46 & 11,0 & 0,34 & 13,0 & - \\ \text { Mo.76-1 } & 49,0 & 19,5 & 0,40 & 18,1 & 0,37 & 12,0 & 20,0 \\ - & 40,0 & 16,3 & 0,41 & 14,7 & 0,37 & 13,0 & - \\ \text { Mo.76-2 } & 35,0 & 15,0 & 0,42 & 12,2 & 0,35 & 13,0 & 20,0 \\ \text { Mo.76-3 } & 37,0 & 19,5 & 0,42 & 13,3 & 0,35 & 13,0 & 17,0 \\ - & 30,0 & 12,5 & 0,42 & 10,5 & 0,35 & 14,0 & - \\ \text { Mo.78-1 } & 49,0 & 19,5 & 0,40 & 17,7 & 0,36 & 12,5 & 20,0 \\ & & & & & & & \\ \text { Mo.81-1 } & 54,0 & 22,0 & 0,40 & 19,5 & 0,36 & 11,5 & 20,0 \\ \text { Mo.88-1 } & 36,0 & 13,6 & 0,38 & 13,3 & 0,37 & 12,0 & 19,0 \\ - & 30,0 & 11,5 & 0,38 & 11,1 & 0,37 & 13,5 & -\end{array}$

\section{Diagnosis}

Conchas de tamaño medio, evolutas con ombligo amplio y somero. Sección subtriangular con región ventral recorrida por una carena bien desarrollada. Costillas primarias radiales, tubérculos poco marcados en el punto de división de las costillas, costillas secundarias anchas y ligeramente retroversas.

Medium-sized, evolute shells, with wide and shallow umbilicus. Subtriangular whorl section with elevated keel. Radial primary ribs, scarcely developed lateral tubercles and slightly retroverse broad secondary ribs.

\section{Descripción}

Formas discoidales de tamaño medio, evolutas, con ombligo amplio y poco profundo. La sección es subtriangular con flancos convexos y región ventral que presenta una carena alta. En las vueltas internas dominan las costillas simples radiales. En la penúltima vuelta las costillas son más anchas que los espacios intercostales y presentan engrosamientos tuberculiformes, a veces verdaderos tubérculos, en el tercio inferior del flanco. A partir de los engrosamientos, las costillas, bien permanecen simples, se bifurcan, o aparecen además costillas intercalares más anchas que los espacios intercostales. El recorrido de las 
costillas es radial o ligeramente retroverso, terminando en una ligera proyección hacia delante en las proximidades de la carena. Los ejemplares disponibles no conservan ni la línea de sutura ni el peristoma.

\section{Discusión}

E. (Eudmetoceras) subbeticum $\mathrm{n}$ sp. es una especie parecida a $E$. (E.) eudmetum Buckman, especie tipo de Eudmetoceras, pero la especie de Buckman se diferencia por ser ligeramente más involuta, por su mayor tamaño al mismo desarrollo ontogenético, por su sección más triangular y por la ornamentación más acusada con tubérculos bien marcados.

E. (E.) subbeticum n sp. presenta también bastante parecido con E. (E.) masticonensis Elmi. La diferencia esencial entre ambas especies estriba en el mayor tamaño de E. (E.) masticonensis para el mismo estadio ontogenético y la existencia de tubérculos bien desarrollados en las vueltas internas del mismo, no existentes o poco marcados en $E$. (E.) subbeticum n. sp.

\section{Eudmetoceras (Eudmetoceras) sp. 1. aff. E. (E.) subbeticum n. sp. [M]}

Fig. $3 f-h$

Material: Mo.73-3 y Mo.76-4, moldes internos casi completos y relativamente bien conservados, Mo.78-2, molde interno incompleto y aplastado, Mo.87-3, molde externo casi completo.

\begin{tabular}{|c|c|c|c|c|c|c|c|}
\hline \multicolumn{8}{|c|}{ Dimensiones en mm } \\
\hline ejemplar & D & $\mathbf{O}$ & O/D & $\mathbf{H}$ & H/D & $\mathrm{N} 1 / 2$ & $\mathrm{~N} 2 / 2$ \\
\hline M0.73-3 & 44,0 & 14,5 & 0,33 & 17,2 & 0,39 & 12,0 & 20,0 \\
\hline - & 34,0 & 11,5 & 0,34 & 13,5 & 0,40 & 一 & 一 \\
\hline Mo.76-4 & 56,0 & 18,5 & 0,32 & 22,5 & 0,40 & 12,0 & 24,0 \\
\hline 一 & 44,0 & 15,5 & 0,35 & 17,3 & 0,39 & 12,0 & 一 \\
\hline Mo.78-2 & 42,0 & 14,0 & 0,33 & 17,2 & 0,41 & 11,0 & - \\
\hline Mo.87-3 & 41,0 & 14,0 & 0,34 & 16,5 & 0,40 & 12,0 & 19,0 \\
\hline- & 32,0 & 11,2 & 0,35 & 12,5 & 0,39 & 13,0 & 一 \\
\hline
\end{tabular}

\section{Discusión}

Estos ejemplares, también recogidos en la sección del Despeñadero, son bastante parecidos a $E$. (E.) subbeticum n. sp., pero se diferencian del mismo por ser más involutos, por tener los flancos muy planos, las costillas primarias algo más cortas y terminadas en un engrosamiento en el que se bifurcan o trifurcan en otras igualmente gruesas y de recorrido retroverso que terminan en una carena bien marcada. El ombligo es poco profundo con reborde umbilical redondeado en las vueltas internas y abrupto en las vueltas externas. No se observa la línea de sutura. Ninguno de los ejemplares ha sido recogido "in situ" pero uno de ellos aparece asociado con un fragmento de Haplopleuroceras eximium Gerard. Esto es indicativo de que estratigráficamente se encuentran en el Aaleniense superior (Zona de
Concavum, Subzona de Limitatum) o en el Bajociense inferior (Zona de Discites, Subzona de Walkeri).

\section{CONCLUSIONES}

El análisis de los hammatocerátidos recogidos en Aaleniense superior (Zona de Concavum) y Bajociense inferior (Zona de Discites) del Subbético muestra formas pertenecientes al género Eudmetoceras que pertenecen a especies hasta ahora no descritas.

Se describe una nueva especie, E. (Eudmetoceras) subbeticum n. sp. Esta nueva especie y otras formas próximas, E. (Eudmetoceras) sp. 1. aff. E. (E.) subbeticum, aparecen en facies hemipelágicas (margas y margocalizas) del surco del dominio Subbético Medio.

$E$. (E.) subbeticum n. sp. comprende formas de tamaño más reducido, para el mismo desarrollo ontogenético, y con ornamentación, especialmente tubérculos, menos desarrollada que otras especies congéneres. Estos caracteres, tamaño reducido y ornamentación poco marcada, separan generalmente las formas de cuencas hemipelágicas, como es el caso de las subbéticas, de las de plataformas epicontinentales, de las que procecen los tipos de las demás especies de Eudmetoceras hasta ahora descritas.

\section{AGRADECIMIENTOS}

La autora quiere expresar su agadecimiento más sincero a Gemma Martínez y a Sixto Fernández López (Universidad Complutense de Madrid) por la lectura minuciosa y crítica del manuscrito; sus agradables sugerencias han mejorado considerablemente el contenido final del mismo. Este trabajo forma parte de los resultados obtenidos con el Proyecto de Investigación PB97-0826, financiado por la DGESIC (Dirección General de Enseñanza Superior e Investigación Científica).

\section{REFERENCIAS}

Bayle, E. 1878. Fossiles principaux des terrains. Mémoire de la Carte géologique de France, 4, 157 lám.

Buckman, S.S. 1887-1907. A monograph of the ammonites of the Inferior Oolite Series. Monographs of the Palaeontological Society of London, $456+103 \mathrm{pp}$ (1887-1894) and Supplement (1898-1907) $24+262$ pp.

Buckman, S.S. 1909-1930. Type Ammonites. London, Weldon and Wesley. Volumes 1-7, 790 pls. Reprint (1972-1976), Historia Naturalis Classica, 93: no 1. Cramer Verlag and Weldon and Wesley, Lehre and Codicote.

Elmi, S. 1963. Les Hammatoceratinae (Ammonitina) dans le Dogger Inférieur du Bassin Rhodanien. Travaux du labotoire de Geológie de la Faculté des Sciences de Lyon, N. S. 10, 1-121.

García-Dueñas, V., Linares A. y Mouterde, R. 1967. Datos estratigráficos sobre la serie mesozoica del Río de las 
Juntas, Montillana, Zona Subbética (Granada). Acta Geológica Hispánica, 3, 65-68.

Hyatt, A. 1867. The fossil cephalopods of the Museum of Comparative Zoology. Bulletin of the Museum of Comparative Zoology, 5, 71-102.

Hyatt, A. 1889. Genesis of the Arietitidae. Smithsonian Contribution to Knowledge, 673, Washington, xi +238 pp.

Jaworski, E. 1926. La fauna del Lías y Dogger de la Cordillera en la parte meridional de la provincia de Mendoza. Acta Nacional de la Academia de Ciencias Naturales, 9, 138-319.

Linares, A. y Sandoval, J. 1988. Asthenoceras y Fontannesia (Grammoceratinae, Ammonitina) del tránsito Aalenense-Bajocense de la Zona Subbética. Revista Española de Paleontología, 3, 3-11.

Linares A. y Sandoval J. 1993. El Aaleniense de la Cordillera Bética (Sur de España), Análisis bioestratigráfico y caracterización paleobiogeográfica. Revista de la Sociedad Geológica de España, 6, 177 206.

Linares A. and Sandoval J. 1996. The genus Haplopleuroceras (Erycitidae, Ammonitina) in the Betic Cordillera, Southern Spain. Geobios, 29, 287-305.

Martínez G. 1992. Hammatoceratinae (Ammonitina) del Toarciense superior y Aaleniense en la Cordillera Ibérica. Tesis doctoral, Univ. Complutense Madrid, 331 pp.

Martínez G. 1996. Los Hammatoceratinae (Ammonitina) del Toarciense y Aaleniense en la Cordillera Ibérica y Cantábrica oriental. Implicaciones paleobiogeográficas.
Revista Española de Paleontología, $\mathbf{N}^{0}$ Extraordinario, 114-121.

Renz, C. 1925. Beiträge zur Cephalopodenfauna des älteren Doggers am Monte San Giuliano (Monte Erice) bei Trapani in Westsizilien. Abhandlungen der Schweizerischen Palaeontologischen Gesellschaft, 45, 1-33.

Sadki, D. 1996. Le Haut-Atlas Central (Maroc). Stratigraphie et paléontologie du Lias Supérieur et du Dogger Inférieur. Dynamique du Bassin et des peuplements. Documents des Laboratoires de Géologe Lyon, 142, 1-245.

Sandoval, J. and Chandler, R.B. 2000. The Sonninniid ammonite Euhoploceras from the Middle Jurassic of south-west England and southern Spain. Palaeontology, 43, 495-533.

Sandoval, J., Linares, A. and Henriques, M.H. 2001. The Middle Jurassic genus Riccardiceras (Otoitidae, Ammonitina) in the Westernmost Tethys, Betic Cordillera and Lusitanian Basin. Revue de Paléobiologie, 8, 29-44.

Westermann, G. E. G. 1964. The Ammonite Fauna of the Kialagvik Formation at Wide Bay, Alaska Peninsula, Part I, Lower Bajocian (Aalenian). Bulletin of American Palaeontology, 47, 329-503.

Westermann, G. E. G. and Riccardi, A. C. 1972. Middle Jurassic ammonoids fauna and biochronology of the Argentine-Chilean Andes. Part. I, Hildocerataceae. Palaeontographica Abteilung A, 140, 1-116.

Zittel, K. A. von 1884. Grundzüge der Palaeontologie (Palaeozoologie). R. Oldenbour, Munich-Leipzig, 971 pp.

Manuscrito recibido: 18 de diciembre, 2001 Manuscrito aceptado: 24 de abril, 2002 